\title{
Proteomic inventory of myocardial proteins from patients with chronic Chagas' cardiomyopathy
}

\author{
P.C. Teixeira ${ }^{1,3,4}$, L.K. Iwai ${ }^{1,4}$, \\ A.C.K. Kuramoto ${ }^{1,4}$, \\ R. Honorato ${ }^{2}$, A. Fiorelli ${ }^{2}$, \\ N. Stolf ${ }^{2}$, J. Kalil1,3,4 \\ and E. Cunha-Neto ${ }^{1,3,4}$
}

\author{
${ }^{1}$ Laboratório de Imunologia, 2Divisão de Cirurgia Torácica, Instituto do Coração, \\ Hospital das Clínicas, ${ }^{3}$ Disciplina de Imunologia Clínica e Alergia, \\ Faculdade de Medicina, Universidade de São Paulo, São Paulo, SP, Brasil \\ ${ }^{4}$ Instituto de Investigação em Imunologia, Instituto do Milênio, CNPq/MCT, \\ São Paulo, SP, Brasil
}

Correspondence

E. Cunha-Neto

Laboratório de Imunologia

InCor-HC-FM, USP

Av. Dr. Eneas C. Aguiar, 44

Bloco II, 9 andar

05403-000 São Paulo, SP

Brasil

Fax: +55-11-3069-5953

E-mail: edecunha@gmail.com

Research supported by FAPESP (Nos. 00/14549-4 and 01/00729-3). P.C. Teixeira is supported by a FAPESP grant (No. 04/15322-4), and

E. Cunha-Neto and J. Kalil are recipients of productivity awards from CNPq (Nos. 302970-2004-5 and 307541-2003-7).

Received December 20, 2005 Accepted August 23, 2006

\begin{abstract}
Chronic Chagas' disease cardiomyopathy (CCC) is an often fatal outcome of Trypanosoma cruzi infection, with a poorer prognosis than other cardiomyopathies. CCC is refractory to heart failure treatments, and is the major indication of heart transplantation in Latin America. A diffuse myocarditis, plus intense myocardial hypertrophy, damage and fibrosis, in the presence of very few $T$. cruzi forms, are the histopathological hallmarks of CCC. To gain a better understanding of the pathophysiology of CCC, we analyzed the protein profile in the affected CCC myocardium. Homogenates from left ventricular myocardial samples of end-stage CCC hearts explanted during heart transplantation were subjected to two-dimensional electrophoresis with Coomassie blue staining; protein identification was performed by MALDI-ToF mass spectrometry and peptide mass fingerprinting. The identification of selected proteins was confirmed by immunoblotting. We demonstrated that 246 proteins matched in gels from two CCC patients. They corresponded to 112 distinct proteins. Along with structural/contractile and metabolism proteins, we also identified proteins involved in apoptosis (caspase 8, caspase 2), immune system ( $\mathrm{T}$ cell receptor $\beta$ chain, granzyme A, HLA class I) and stress processes (heat shock proteins, superoxide dismutases, and other oxidative stress proteins). Proteins involved in cell signaling and transcriptional factors were also identified. The identification of caspases and oxidative stress proteins suggests the occurrence of active apoptosis and significant oxidative stress in CCC myocardium. These results generated an inventory of myocardial proteins in CCC that should contribute to the generation of hypothesis-driven experiments designed on the basis of the classes of proteins identified here.
\end{abstract}

Key words - Chagas' disease - Cardiomyopathy

- Proteomic analysis

- Two-dimensional electrophoresis

- MALDI-ToF 


\section{Introduction}

Chagas' disease is a significant cause of morbidity and mortality in Central and South America, affecting about 13 million people (1). The disease is caused by infection with the intracellular protozoan parasite Trypanosoma cruzi. About 30\% of Chagas' disease patients develop chronic Chagas' disease cardiomyopathy (CCC), an inflammatory cardiomyopathy that occurs decades after the initial infection, and one-third progress further to a particularly aggressive, life-threatening dilated cardiomyopathy. In spite of the recent advances in vector control, the millions of patients already infected deserve more attention from the scientific community. Furthermore, clinical progression, length of survival and overall prognosis are significantly worse in CCC patients compared with patients with dilated cardiomyopathy of non-inflammatory etiology $(2,3)$.

CCC heart lesions show histopathological findings consistent with inflammation and a myocardial remodeling process: T cell/ macrophage-rich myocarditis, hypertrophy, and fibrosis with heart fiber damage (4). The local cytokine production profile is consistent with a T1-type response, with interferongamma $(\gamma)$-induced chemokines (5-12). Gene expression profiling of CCC myocardial tissues showed that $15 \%$ of the known genes selectively up-regulated in CCC are IFN- $\gamma$ inducible (12). Exposure of cardiomyocytes to IFN- $\gamma$ can up-regulate the expression of atrial natriuretic factor (12), suggesting that IFN- $\gamma$ may directly modulate gene expression in myocardial cells. These results are consistent with a possible role of IFN- $\gamma$ induced chronic inflammation in modulating myocardial gene expression.

However, gene expression profiling may not be faithfully reflected at the protein level. Advances in two-dimensional (2-D) electrophoresis, mass spectrometry, and bioinformatics, along with progress in genomic sequence analysis now make possible a direct evaluation of large-scale protein profiles (13).

In order to study the global protein profile of the myocardium under the effect of chronic inflammation in CCC heart tissue, and to gain insight about the pathophysiology of CCC, we analyzed the myocardial proteome from end-stage CCC patients.

\section{Patients, Material and Methods}

\section{Reagents}

Immobilized $\mathrm{pH}$ gradient gel buffer, tryp$\sin$ (sequencing grade), $\alpha$-cyano-4-hydroxycinnamic acid and other analytical grade reagents were purchased from Amersham Biosciences (Uppsala, Sweden), with the exception of ammonium bicarbonate, analytical grade acetonitrile and trifluoroacetic acid (TFA) that were obtained from Merck (Darmstadt, Germany). ACTH peptide 1839 and (Ile7)-angiotensin III were obtained from Sigma (St. Louis, MO, USA). All stock solutions were prepared with deionized water using a Milli-Q Academic System (Millipore Co., Billerica, MA, USA).

\section{Sample preparation}

The protocol was approved by the Institutional Review Board of the University of São Paulo School of Medicine and written informed consent was obtained from the patients.

Myocardial left ventricular free wall heart samples were obtained from two end-stage heart failure CCC patients with a diagnosis established by seropositivity to T. cruzi in at least two of three tests, i.e., ELISA, indirect immunofluorescence and indirect hemagglutination, and by positive epidemiology. Both patients were female and were 42 and 62 years old, with an ejection fraction $<40 \%$. The hearts were explanted on the occasion of heart transplantation at the Heart Institute - InCor, University of São Paulo School of Medicine, São Paulo, SP, Brazil. Samples 
were dissected, frozen in liquid nitrogen and stored at $-80^{\circ} \mathrm{C}$. The tissue $(100 \mathrm{mg})$ was homogenized in $1 \%$ SDS (w/v) and $0.5 \mathrm{mM}$ TLCK (1 mL), submitted to three sonication cycles and centrifuged at $12,000 \mathrm{~g}$ for 15 $\min$ at $4^{\circ} \mathrm{C}(14)$. The samples were passed through Millipore Centricon YM-3 filters (molecular mass cutoff at $3000 \mathrm{Da}$ ) and the protein content of the supernatant solution of each extract was quantified by the bicinchoninic acid method (15).

\section{Two-dimensional gel electrophoresis}

Isoelectric focusing was carried out on 24-cm long immobilized $\mathrm{pH}$ gradient gel strips, with a $\mathrm{pH}$ range of 3.0 to 10.0 (Im mobiline DryStrip gels, Amersham Biosciences). The strips were rehydrated in $8 \mathrm{M}$ urea, $0.5 \%$ CHAPS, $0.5 \%$ IPG buffer, $0.2 \%$ DTT, and traces of bromophenol blue containing $1 \mathrm{mg}$ of cardiac protein extract for 12 $\mathrm{h}$ at $20^{\circ} \mathrm{C}$. Isoelectric focusing was performed using the Ettan IPGphor Isoelectric Focusing System (Amersham Biosciences) at $20^{\circ} \mathrm{C}$ at $50 \mathrm{~mA}$ /strip in an increasing voltage gradient $(500 \mathrm{~V}$ for $1 \mathrm{~h}, 1000 \mathrm{~V}$ for $1 \mathrm{~h}$, and $8000 \mathrm{~V}$ for $8 \mathrm{~h}$, accumulating a total of $64,000 \mathrm{Vh}$ ), according to manufacturer instructions. Before the second dimension electrophoresis, the strips were equilibrated twice for $15 \mathrm{~min}$ in a buffer containing $50 \mathrm{mM}$ Tris, $\mathrm{pH} 8.8,6 \mathrm{M}$ urea, 30\% glycerol (v/v), $2 \%$ SDS (w/v), $0.002 \%$ bromophenol blue $(\mathrm{w} / \mathrm{v})$, and in the presence of $10 \mathrm{mg} / \mathrm{mL}$ DTT in the first step and $25 \mathrm{mg} / \mathrm{mL}$ iodoacetamide in the second step. The second dimension was carried out on a vertical DaltSix system (Amersham Biosciences) at $20^{\circ} \mathrm{C}$ on $12.5 \%$ polyacrylamide gels $(24 \times 18 \mathrm{~cm})$ for $5 \mathrm{~h}$ at $100 \mathrm{~W}$. Coomassie blue-stained gels were digitized with an ImageScanner and analyzed with the ImageMaster 2D Elite 3.10 software (Amersham Biosciences). The matching spots in the two samples were selected and the volume (related to the quantity of protein) of each spot was normalized on the basis of total volume of all spots in each gel.

\section{Protein digestion}

All spots visualized in each gel were excised, subjected to tryptic digestion and processed in a robotic workstation (Ettan Spot Handling Workstation, Amersham Biosciences). Briefly, spots (1.4 $\mathrm{mm}$ in diameter) were excised from the gel and then washed in $50 \mathrm{mM}$ ammonium bicarbonate $\left(\mathrm{NH}_{4} \mathrm{HCO}_{3}\right)$ in $50 \%$ acetonitrile (ACN) until complete destaining and SDS removal and then dried. Dehydrated gel plugs were rehydrated with $30 \mu \mathrm{L}$ of a solution containing $0.3 \mu \mathrm{g}$ trypsin in $20 \mathrm{mM} \mathrm{NH}_{4} \mathrm{HCO}_{3}$. Digestion was performed at $37^{\circ} \mathrm{C}$ for $6 \mathrm{~h}$. Digested peptides were extracted by the addition of 50 $\mu \mathrm{L} \mathrm{50 \%} \mathrm{ACN/0.5 \%} \mathrm{TFA} \mathrm{at} \mathrm{room} \mathrm{tempera-}$ ture for $1 \mathrm{~h}$. The procedure was repeated and the supernatants were combined. Samples were concentrated and resuspended in $5 \mu \mathrm{L}$ $5 \% \mathrm{ACN} / 2.5 \%$ TFA.

\section{Protein identification}

Tryptic fragments were analyzed with the Ettan MALDI-ToF Pro mass spectrometer (Amersham Biosciences). For peptide mass fingerprinting (PMF) analysis, a small quantity of each processed sample $(0.5 \mu \mathrm{L})$ corresponding to a spot was applied on a metal slide mixed with the same quantity of matrix ( $\alpha$-cyano-4-hydroxycinnamic acid) and taken to the mass spectrometer for analysis. To ensure maximal accuracy, trypsin autolysis peptides (842.51 and 2211.10 Da) were used for internal mass calibration and (Ile7)-angiotensin III (897.53 Da) and ACTH peptide 18-39 (2465.20 Da) were used for external calibration of the instrument. The $\mathrm{m} / \mathrm{z}$ values for trypsin autolysis peptides and for contaminants $(842.5,1045.8,1066.4$, 2211.1, 2225.1, and 2299.2) were excluded from the list of tryptic peptides representing the PMF of proteins. 
Proteins were identified by comparing the PMF of each spot to virtual tryptic digestion of proteins using the MALDI-ToF Evaluation software (Amersham Biosciences) and the non-redundant NCBI databank (H. sapiens), using as search parameters 0.2-Da peptide mass tolerance and 1 maximum missed cleavage sites. The mass lists of each spectrum were also submitted to the Mascot tool (http://www.matrixscience.com) and SwissProt databank (H. sapiens) with mass tolerance of $0.5 \mathrm{Da}$ and 1 maximum missed cleavage as parameters. The only allowed post-translational modification was oxidation. We considered expectation values $\leq 0.1$ as significant protein identifications. After protein identification in both gels, a composite image of the two gels was constructed by matching the spots. We could enumerate the protein spots that were matched and identified as the same protein in the two gels, as well as those that were only identified in one of the gels. The functional classification of the proteins identified in both gels was adapted from the Gene Ontology classification using the FatiGO tool (http://fatigo. bioinfo.cipf.es).

\section{Western blot analysis}

Cardiac tissue homogenates in SDSPAGE sample buffer were heated $\left(95^{\circ} \mathrm{C}, 5\right.$ min), run on SDS-polyacrylamide gels and then blotted onto nitrocellulose membranes. Next, the membranes were incubated with monoclonal antibodies against heat shock protein 60, protein disulfide isomerase, $\mathrm{Cu} /$ Zn superoxide dismutase, caspase 8 (Stressgen Bioreagents, Victoria, British Columbia, Canada) and caspase 2 (rabbit polyclonal antibody). Antibodies were kindly provided by Drs. Celio X. Santos, Francisco Laurindo, and Gustavo Amarante-Mendes (University of São Paulo). After incubation with the appropriate horseradish peroxidasecoupled conjugate, blots were developed by chemiluminescence using ECL Plus West- ern Blotting Detection Reagents (Amersham Biosciences, Little Chalfont, Buckinghamshire, UK).

\section{Results}

Two-dimensional electrophoresis of the myocardium from CCC patients allowed us to observe ca. 400 spots on each gel (Figure 1). Using MALDI-ToF analysis with SWISSPROT and the non-redundant NCBI databases, we identified proteins in 316 spots on the gel from patient 1 and 259 spots on the gel from patient 2, 246 of which were matched on both gels. Of the identified proteins, 112 were distinct proteins (Table 1). A representative spectrum of the tryptic fragments of a protein is shown in Figure 2, illustrating the mass/charge values from the trypsin autolysis products.

Table 2 shows the distribution of the 112 distinct proteins among functional categories according to the Gene Ontology classification (http://fatigo. bioinfo.cipf.es). As expected, proteins of the contractile apparatus and involved in energy generation were among the most abundant groups in CCC heart tissue. This analysis was able to identify less abundantly expressed proteins, such as transcriptional factors and proteins produced by the inflammatory infiltrate and related to the apoptotic process.

Structural proteins (19\% of all distinct proteins) included multiple constituents of the sarcomeric actomyosin complex (15 proteins) such as heavy and light chains of cardiac myosin, several isoforms of actin, troponin and tropomyosin, actin-binding and Z-band proteins, as well as cytoskeletal proteins (6 proteins). The atrial isoform of myosin light chain (MYL4), which is normally suppressed after birth (16), was identified in the ventricular CCC samples. We identified six spots corresponding to vimentin, which is expressed by fibroblasts, suggesting the occurrence of a fibrotic process, a hallmark of CCC myocardium (17).

More than $38 \%$ of all distinct proteins 

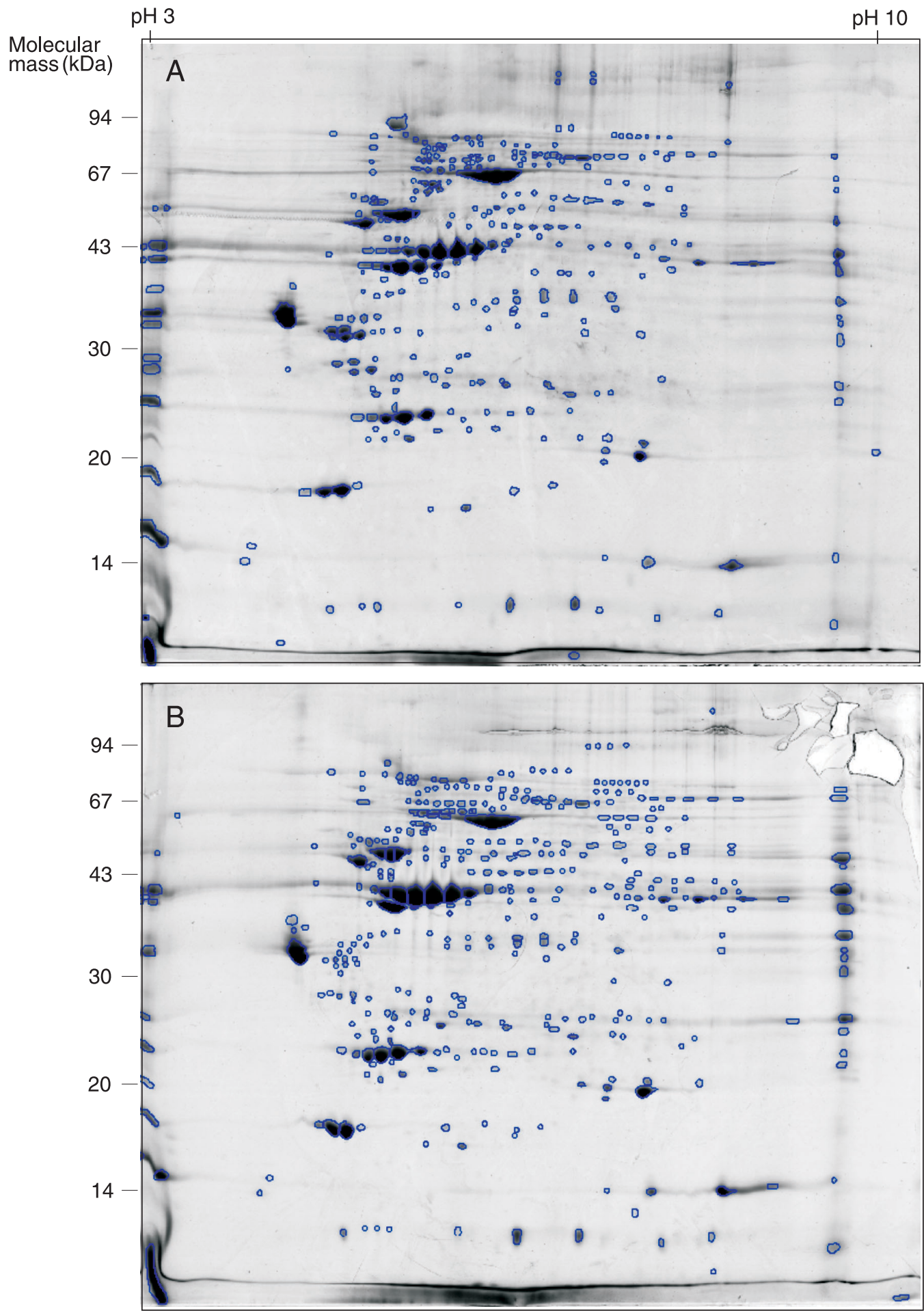

Figure 1. Two-dimensional gel electrophoresis of proteins in the myocardium of patients with chronic Chagas' disease cardiopathy (CCC). $A$ and B, Samples from CCC patients \#1 and \#2; the outlines of spots that were selected for protein identification are shown. Myocardium proteins were separated by isoelectric focusing on 24-cm long immobilized $\mathrm{pH}$ gradient gel strips containing a linear 3-10 pH gradient, followed by SDS-PAGE on vertical $12.5 \%$ gels. Proteins were detected by Coomassie blue R-250 staining. 
Table 1. Proteins identified in the myocardium of patients with chronic Chagas' disease cardiopathy by 2-D gel electrophoresis-peptide mass fingerprinting analysis.

\begin{tabular}{|c|c|c|c|c|c|c|c|c|c|c|c|}
\hline Symbol & Description & Exp. ${ }^{1}$ & $\begin{array}{c}\text { Cov. }^{2} \\
(\%)\end{array}$ & $\begin{array}{l}\mathrm{pl} \\
(\mathrm{T})\end{array}$ & $\begin{array}{l}\mathrm{pl} \\
(\mathrm{E})\end{array}$ & $\begin{array}{l}\text { Mass } \\
(\mathrm{T})\end{array}$ & $\begin{array}{l}\text { Mass } \\
(\mathrm{E})\end{array}$ & $\begin{array}{l}\text { N. } \\
\text { Vol. }^{3}\end{array}$ & $\begin{array}{l}\text { Accession } \\
\text { number }\end{array}$ & $\begin{array}{l}\text { No. } \\
\text { Spots }^{4}\end{array}$ & $\begin{array}{c}\text { T. } \\
\text { Vol. } 5\end{array}$ \\
\hline \multicolumn{12}{|c|}{ 1. Structural and Contractile Proteins } \\
\hline ACTA1 & actin, $\alpha 1$, skeletal muscle & 0.027 & 30.2 & 5.2 & 5.5 & 42.3 & 51.6 & 0.196 & P68133 & 3 & 0.20 \\
\hline АСТА2 & actin, $\alpha 2$, smooth muscle, aorta & 0.010 & 19.7 & 5.3 & 4.8 & 42.3 & 42.1 & 0.034 & P62736 & 15 & 0.03 \\
\hline ACTC & actin, $\alpha$, cardiac muscle & 0.006 & 28.1 & 5.2 & 3.0 & 42.3 & 56.0 & 0.816 & P68032 & 14 & 0.82 \\
\hline ACTG2 & actin, $\gamma 2$, smooth muscle, enteric & 0.018 & 34.3 & 5.3 & 5.8 & 42.3 & 54.7 & 3.618 & P63267 & 8 & 3.62 \\
\hline ACTN2 & actinin, $\alpha 2$ & 0.018 & 20.9 & 5.3 & 5.7 & 104.4 & 91.3 & 0.165 & P35609 & 8 & 0.17 \\
\hline DES & desmin & 0.000 & 41.7 & 5.2 & 5.2 & 53.6 & 65.3 & 1.506 & $\mathrm{P} 17661$ & 13 & 1.51 \\
\hline KIAA0992 & palladin & 0.083 & 12.4 & 9.3 & 9.0 & 45.6 & 31.0 & 0.118 & Q7Z3W0 & 1 & 0.12 \\
\hline LMNA & lamin A/C (70-kDa lamin) & 0.010 & 15.0 & 6.3 & 8.4 & 65.0 & 66.2 & 0.037 & P02545 & 2 & 0.06 \\
\hline MYBPC3 & myosin-binding protein $\mathrm{C}$, cardiac & 0.001 & 8.8 & 6.2 & 7.5 & 141.8 & 107.2 & 0.030 & Q14896 & 4 & 0.12 \\
\hline MYH6 & $\begin{array}{l}\text { myosin, heavy polypeptide } 6 \\
\text { cardiac muscle, } \alpha\end{array}$ & 0.001 & 6.0 & 5.9 & 8.3 & 223.5 & 125.0 & 0.041 & P13533 & 1 & 0.04 \\
\hline MYH7 & $\begin{array}{l}\text { myosin, heavy polypeptide } 7 \text {, } \\
\text { cardiac muscle, } B\end{array}$ & 0.000 & 33.6 & 5.3 & 5.6 & 183.1 & 75.0 & 0.115 & P12883 & 12 & 0.79 \\
\hline MYL2 & $\begin{array}{l}\text { myosin, light polypeptide } 2 \text {, } \\
\text { regulatory, cardiac, slow }\end{array}$ & 0.000 & 69.3 & 4.9 & 4.8 & 18.8 & 18.9 & 2.749 & P10916 & 3 & 4.78 \\
\hline MYL3 & $\begin{array}{l}\text { myosin, light polypeptide } 3 \text {, alkali, } \\
\text { ventricular, skeletal, slow }\end{array}$ & 0.000 & 56.2 & 5.0 & 5.3 & 22.0 & 26.9 & 2.636 & P08590 & 10 & 7.54 \\
\hline MYL4 & $\begin{array}{l}\text { myosin, light polypeptide } 4 \text {, alkali, } \\
\text { atrial, embryonic }\end{array}$ & 0.010 & 36.0 & 5.0 & 5.3 & 21.4 & 30.0 & 0.021 & P12829 & 2 & 0.06 \\
\hline MYOZ2 & myozenin 2 & 0.014 & 19.7 & 7.9 & 6.4 & 29.9 & 42.1 & 0.016 & Q9NPC6 & 2 & 0.04 \\
\hline RTN2 & reticulon 2 & 0.164 & 16.4 & 5.3 & 9.5 & 33.4 & 41.3 & 0.156 & O75298 & 1 & 0.16 \\
\hline SSH3 & slingshot homolog 3 & 0.059 & 22.0 & 9.5 & 5.3 & 16.5 & 25.8 & 0.017 & Q8TE77 & 1 & 0.02 \\
\hline TNNT2 & troponin T2, cardiac & 0.010 & 20.1 & 4.9 & 5.3 & 33.0 & 51.8 & 4.163 & P45379 & 3 & 4.49 \\
\hline TPM1 & tropomyosin $1(\alpha)$ & 0.014 & 34.9 & 4.6 & 4.4 & 26.6 & 42.0 & 5.375 & P09493 & 2 & 5.59 \\
\hline TUBB & tubulin, B polypeptide & 0.019 & 19.9 & 4.8 & 5.2 & 30.6 & 69.5 & 0.066 & P07437 & 1 & 0.07 \\
\hline VIM & vimentin & 0.004 & 32.2 & 5.1 & 5.0 & 53.7 & 62.1 & 0.066 & P08670 & 6 & 0.46 \\
\hline
\end{tabular}

\section{Metabolism}

2.1. Glycolysis

ALDOA fructose 1,6-biphosphate aldolase

ENO1 enolase 1

GAPDH glyceraldehyde-3-phosphate dehydrogenase

LDHB lactate dehydrogenase $B$

PDHB pyruvate dehydrogenase, E1 beta

$\begin{array}{rrrrrrrrrr}0.020 & 30.0 & 8.8 & 9.5 & 39.7 & 51.3 & 0.821 & \mathrm{P} 04075 & 1 & 0.82 \\ 0.026 & 24.4 & 7.0 & 7.6 & 47.5 & 60.2 & 0.023 & \mathrm{P} 06733 & 1 & 0.02 \\ 0.019 & 15.2 & 8.7 & 9.5 & 36.2 & 45.6 & 0.665 & \mathrm{P} 04406 & 1 & 0.66 \\ & & & & & & & & & \\ 0.013 & 15.9 & 5.7 & 6.7 & 36.8 & 45.5 & 0.103 & \mathrm{P} 07195 & 3 & 0.31 \\ 0.000 & 26.0 & 5.4 & 6.0 & 36.8 & 43.2 & 0.038 & \mathrm{P} 11177 & 2 & 0.07 \\ & & & & & & & & & \\ 0.006 & 10.0 & 7.4 & 7.7 & 86.2 & 84.3 & 0.046 & \mathrm{Q} 99798 & 7 & 0.62 \\ 0.025 & 22.0 & 7.8 & 8.0 & 54.7 & 66.3 & 0.051 & \mathrm{P} 09622 & 3 & 0.18 \\ & & & & & & & & & \\ 0.068 & 11.0 & 9.4 & 6.1 & 49.0 & 64.4 & 0.059 & \mathrm{P} 36957 & 2 & 0.12 \\ 0.018 & 23.5 & 9.0 & 8.1 & 54.8 & 58.3 & 0.058 & \mathrm{P} 07954 & 4 & 0.17 \\ 0.099 & 23.7 & 9.0 & 9.5 & 33.7 & 42.7 & 0.103 & \mathrm{P} 40926 & 1 & 0.10 \\ 0.015 & 9.3 & 6.6 & 7.3 & 114.7 & 90.4 & 0.027 & \mathrm{Q} 02218 & 6 & 0.15 \\ 0.005 & 14.5 & 7.1 & 7.5 & 73.7 & 77.4 & 0.080 & \mathrm{P} 31040 & 4 & 0.30\end{array}$

2.2. Tricarboxylic Acid Cycle

ACO2 aconitase 2, mitochondrial

DLD dihydrolipoamide dehydrogenase precursor

DLST dihydrolipoamide succinyltransferase

$\mathrm{FH} \quad$ fumarate hydratase precursor

$\mathrm{MDH} 2$ malate dehydrogenase 2, mitochondria

$\mathrm{OGDH}$ oxoglutarate dehydrogenase (lipoamide)

SDHA succinate dehydrogenase complex,

subunit $A$, flavoprotein $(F p)$

2.3. Oxidative Phosphorylation and Electron Transport

\begin{tabular}{|c|c|c|c|c|c|c|c|c|c|c|c|}
\hline ATP5A1 & ATP synthase, F1 complex, $\alpha$ polypeptide 1 & 0.010 & 21.7 & 9.3 & 9.5 & 59.8 & 64.6 & 0.821 & P25705 & 2 & 0.82 \\
\hline ATP5B & ATP synthase, F1 complex, B polypeptide & 0.000 & 46.1 & 5.3 & 5.0 & 56.5 & 63.6 & 1.548 & P06576 & 2 & 1.55 \\
\hline ATP5C1 & ATP synthase, F1 complex, $\gamma$ polypeptide 1 & 0.047 & 26.1 & 9.1 & 9.5 & 32.3 & 38.8 & 0.313 & P36542 & 1 & 0.31 \\
\hline ETFDH & $\begin{array}{l}\text { electron-transferring-flavoprotein } \\
\text { dehydrogenase }\end{array}$ & 0.002 & 10.1 & 6.9 & 7.9 & 65.5 & 73.6 & 0.013 & Q16134 & 3 & 0.07 \\
\hline
\end{tabular}


Table 1 continued.

\begin{tabular}{|c|c|c|c|c|c|c|c|c|c|c|c|}
\hline Symbol & Description & Exp. ${ }^{1}$ & $\begin{array}{c}\text { Cov. }^{2} \\
(\%)\end{array}$ & $\begin{array}{l}\mathrm{pl} \\
(\mathrm{T})\end{array}$ & $\begin{array}{l}\mathrm{pl} \\
(\mathrm{E})\end{array}$ & $\begin{array}{c}\text { Mass } \\
(\mathrm{T})\end{array}$ & $\begin{array}{l}\text { Mass } \\
(\mathrm{E})\end{array}$ & $\begin{array}{l}\text { N. } \\
\text { Vol. }\end{array}$ & $\begin{array}{l}\text { Accession } \\
\text { number }\end{array}$ & $\begin{array}{l}\text { No. } \\
\text { Spots }^{4}\end{array}$ & $\begin{array}{c}\text { T. } \\
\text { Vol. } 5\end{array}$ \\
\hline NDUFS1 & $\begin{array}{l}\text { NADH dehydrogenase (ubiquinone) } \\
\text { Fe-S protein } 1\end{array}$ & 0.016 & 16.5 & 5.9 & 5.8 & 80.5 & 82.5 & 0.114 & P28331 & 6 & 0.42 \\
\hline NDUFS3 & $\begin{array}{l}\text { NADH dehydrogenase (ubiquinone) } \\
\text { Fe-S protein } 3\end{array}$ & 0.012 & 27.7 & 7.0 & 6.0 & 30.3 & 30.9 & 0.035 & O75489 & 2 & 0.06 \\
\hline NDUFS8 & $\begin{array}{l}\text { NADH dehydrogenase (ubiquinone) } \\
\text { Fe-S protein } 8\end{array}$ & 0.091 & 18.1 & 5.5 & 5.1 & 24.3 & 25.6 & 0.028 & O00217 & 1 & 0.03 \\
\hline NDUFV1 & $\begin{array}{l}\text { NADH dehydrogenase (ubiquinone) } \\
\text { flavoprotein } 1\end{array}$ & 0.042 & 8.6 & 9.1 & 9.5 & 50.5 & 62.2 & 0.088 & P49821 & 1 & 0.09 \\
\hline NDUFV2 & $\begin{array}{l}\text { NADH dehydrogenase (ubiquinone) } \\
\text { flavoprotein } 2\end{array}$ & 0.015 & 26.0 & 7.1 & 6.6 & 25.7 & 27.1 & 0.058 & P19404 & 1 & 0.06 \\
\hline UQCRC1 & $\begin{array}{l}\text { ubiquinol-cytochrome c reductase } \\
\text { core protein I }\end{array}$ & 0.000 & 26.6 & 5.9 & 6.4 & 53.3 & 60.3 & 0.104 & P31930 & 5 & 0.41 \\
\hline UQCRFS1 & $\begin{array}{l}\text { ubiquinol-cytochrome c reductase, } \\
\text { Rieske iron-sulfur polypeptide } 1\end{array}$ & 0.034 & 12.4 & 8.9 & 7.0 & 29.9 & 29.0 & 0.128 & P47985 & 1 & 0.13 \\
\hline \multicolumn{12}{|c|}{ 2.4. Energy Transduction } \\
\hline CKM & creatine kinase, $\mathrm{M}$ chain & 0.005 & 38.1 & 6.8 & 8.1 & 43.3 & 53.5 & 0.578 & P06732 & 8 & 0.58 \\
\hline CKMT2 & creatine kinase, sarcomeric mitochondrial & 0.149 & 25.1 & 8.9 & 9.5 & 48.0 & 55.9 & 1.211 & P17540 & 1 & 1.21 \\
\hline \multicolumn{12}{|c|}{ 2.5. Lipid Metabolism/ß-Oxidation } \\
\hline ALG1 & B-1,4 mannosyltransferase & 0.129 & 14.7 & 5.6 & 4.7 & 26.5 & 35.2 & 0.023 & Q9P2Y2 & 1 & 0.02 \\
\hline APOA1 & apolipoprotein A-I & 0.036 & 24.7 & 5.5 & 5.2 & 30.8 & 28.3 & 0.110 & P02647 & 2 & 0.11 \\
\hline $\mathrm{ECH} 1$ & $\begin{array}{l}\text { enoyl co-enzyme } \mathrm{A}(\mathrm{CoA}) \text { hydratase } 1 \text {, } \\
\text { peroxisomal }\end{array}$ & 0.006 & 16.2 & 8.7 & 7.8 & 36.1 & 36.9 & 0.021 & Q13011 & 2 & 0.02 \\
\hline ECHS1 & enoyl CoA hydratase 1, mitochondrial & 0.020 & 21.0 & 8.9 & 6.9 & 31.8 & 31.3 & 0.020 & P30084 & 1 & 0.02 \\
\hline FABP3 & fatty acid-binding protein 3 , muscle and heart & 0.038 & 25.2 & 7.0 & 7.0 & 13.3 & 11.9 & 0.365 & P05413 & 3 & 1.15 \\
\hline HMGCS1 & $\begin{array}{l}\text { hydroxymethylglutaryl-CoA synthase, } \\
\text { cytoplasmic }\end{array}$ & 0.160 & 11.9 & 5.8 & 7.3 & 56.0 & 83.2 & 0.031 & Q01581 & 1 & 0.03 \\
\hline \multicolumn{12}{|c|}{ 2.6. Nucleotide and Nucleic Acid Metabolism } \\
\hline ADSS & adenylsuccinate synthase & 0.043 & 8.0 & 8.8 & 6.0 & 50.2 & 52.7 & 0.034 & P30520 & 1 & 0.03 \\
\hline AK1 & adenylate kinase 1 & 0.084 & 23.0 & 7.8 & 9.5 & 21.9 & 25.5 & 0.121 & P00568 & 1 & 0.12 \\
\hline DPYSL2 & dihydropyrimidinase-like 2 & 0.085 & 12.4 & 6.0 & 7.3 & 62.7 & 74.3 & 0.038 & Q16555 & 1 & 0.04 \\
\hline \multicolumn{12}{|c|}{ 2.7. Other Metabolism Proteins } \\
\hline ALDH2 & aldehyde dehydrogenase, mitochondrial & 0.010 & 0.2 & 6.6 & 6.2 & 56.3 & 65.8 & 0.070 & P05091 & 1 & 0.07 \\
\hline GOLGA1 & Golgi autoantigen (golgin 97) & 0.019 & 12.0 & 5.2 & 5.7 & 88.3 & 88.4 & 0.042 & Q92805 & 1 & 0.04 \\
\hline GOT1 & aspartate aminotransferase 1 & 0.039 & 14.8 & 6.5 & 7.9 & 46.5 & 51.5 & 0.073 & P17174 & 2 & 0.14 \\
\hline NSFL1C & p47 protein & 0.082 & 17.3 & 5.1 & 5.9 & 41.1 & 66.5 & 0.037 & Q9UNZ2 & 2 & 0.07 \\
\hline OXCT1 & 3-oxoacid CoA transferase 1 precursor & 0.002 & 17.1 & 7.2 & 6.9 & 56.6 & 68.7 & 0.072 & P55809 & 2 & 0.18 \\
\hline PSMD11 & proteasome $26 \mathrm{~S}$ non-ATPase subunit 11 & 0.087 & 17.3 & 5.5 & 7.5 & 43.2 & 74.3 & 0.019 & 000231 & 1 & 0.02 \\
\hline PYGB & glycogen phosphorylase, brain form & 0.001 & 15.3 & 6.5 & 7.5 & 99.5 & 86.8 & 0.017 & P11216 & 1 & 0.02 \\
\hline TPI1 & triosephosphate isomerase 1 & 0.054 & 22.2 & 6.5 & 8.1 & 26.8 & 28.7 & 0.018 & P60174 & 2 & 0.04 \\
\hline \multicolumn{12}{|c|}{ 3. Stress Response } \\
\hline APTX & aprataxin & 0.088 & 27.0 & 9.2 & 5.0 & 13.3 & 12.3 & 0.015 & Q7Z2E3 & 1 & 0.02 \\
\hline CRYAB & crystallin, $\alpha$ B & 0.010 & 44.0 & 6.8 & 7.6 & 20.1 & 22.6 & 1.865 & P02511 & 5 & 1.86 \\
\hline CRYM & crystallin, mu & 0.002 & 10.5 & 5.1 & 5.1 & 33.9 & 45.6 & 0.026 & Q14894 & 3 & 0.03 \\
\hline DNAJC12 & DnaJ (Hsp40) homolog, subfamily C, member 12 & 0.150 & 19.0 & 5.5 & 7.3 & 23.4 & 29.3 & 0.038 & Q9UKB3 & 1 & 0.04 \\
\hline DNAJC13 & $\begin{array}{l}\text { DnaJ (Hsp40) homolog, subfamily C, } \\
\text { member } 13 \text { (RME8) }\end{array}$ & 0.022 & 18.5 & 8.4 & 7.0 & 50.6 & 61.3 & 0.020 & O75165 & 1 & 0.02 \\
\hline GSTA1 & glutathione S-transferase A1 & 0.073 & 31.1 & 5.4 & 6.2 & 23.3 & 26.9 & 0.030 & P08263 & 1 & 0.03 \\
\hline HSPA5 & heat shock 70-kDa protein 5 & 0.035 & 10.0 & 5.1 & 5.0 & 72.3 & 83.0 & 0.147 & P11021 & 1 & 0.15 \\
\hline HSPA8 & heat shock 70-kDa protein 8 isoform 2 & 0.000 & 35.6 & 5.6 & 5.8 & 53.6 & 77.7 & 0.234 & P11142 & 5 & 0.87 \\
\hline HSPA9B & heat shock $70-\mathrm{kDa}$ protein 9B precursor & 0.002 & 30.2 & 6.0 & 5.7 & 74.1 & 79.4 & 0.194 & P38646 & 6 & 0.70 \\
\hline
\end{tabular}


Table 1 continued.

\begin{tabular}{|c|c|c|c|c|c|c|c|c|c|c|c|}
\hline Symbol & Description & Exp. ${ }^{1}$ & $\begin{array}{c}\text { Cov. }^{2} \\
(\%)\end{array}$ & $\begin{array}{l}\mathrm{pl} \\
(\mathrm{T})\end{array}$ & $\begin{array}{l}\mathrm{pl} \\
(\mathrm{E})\end{array}$ & $\begin{array}{l}\text { Mass } \\
(\mathrm{T})\end{array}$ & $\begin{array}{l}\text { Mass } \\
(\mathrm{E})\end{array}$ & $\begin{array}{c}\text { N. } \\
\text { Vol. }^{3}\end{array}$ & $\begin{array}{l}\text { Accession } \\
\text { number }\end{array}$ & $\begin{array}{l}\text { No. } \\
\text { Spots }^{4}\end{array}$ & $\begin{array}{c}\text { T. } \\
\text { Vol. } 5\end{array}$ \\
\hline HSPB1 & heat shock 27-kDa protein 1 & 0.074 & 36.6 & 8.1 & 5.7 & 22.4 & 30.4 & 0.219 & P04792 & 8 & 0.64 \\
\hline HSPB6 & heat shock protein, $\alpha$-crystallin-related, B6 & 0.010 & 23.0 & 6.0 & 6.7 & 17.2 & 19.3 & 0.008 & 014558 & 3 & 0.04 \\
\hline HSPB7 & $\begin{array}{l}\text { heat shock } 27-\mathrm{kDa} \text { protein family, member } 7 \\
\text { (cardiovascular) }\end{array}$ & 0.007 & 32.1 & 5.4 & 5.2 & 15.5 & 12.2 & 0.011 & Q9UBY9 & 1 & 0.01 \\
\hline HSPCA & heat shock $90-k D a$ protein $1, \alpha$ & 0.009 & 13.0 & 4.9 & 5.0 & 84.5 & 88.4 & 0.036 & P07900 & 1 & 0.04 \\
\hline HSPD1 & chaperonin (HSP60) & 0.059 & 16.9 & 5.7 & 5.4 & 61.2 & 72.6 & 0.117 & P10809 & 4 & 0.23 \\
\hline NOR1 & $\begin{array}{l}\text { oxidored-nitro domain-containing protein } \\
\text { isoform } 1\end{array}$ & 0.132 & 20.6 & 9.4 & 2.9 & 26.7 & 53.2 & 0.132 & Q8N7G2 & 1 & 0.13 \\
\hline PDIA3 & protein disulfide isomerase-associated 3 & 0.045 & 15.2 & 6.0 & 6.5 & 57.2 & 69.7 & 0.025 & P30101 & 4 & 0.11 \\
\hline PRDX2 & peroxiredoxin 2 & 0.002 & 28.0 & 5.2 & 5.4 & 18.5 & 24.4 & 0.125 & P32119 & 3 & 0.23 \\
\hline PRDX6 & peroxiredoxin 6 & 0.066 & 17.4 & 6.0 & 6.8 & 25.1 & 30.0 & 0.071 & P30041 & 1 & 0.07 \\
\hline SERPINA1 & $\alpha-1$ antitrypsin & 0.003 & 26.8 & 5.4 & 4.9 & 46.9 & 71.6 & 0.034 & P01009 & 1 & 0.03 \\
\hline SOD1 & superoxide dismutase 1 , soluble & 0.137 & 32.7 & 5.8 & 5.9 & 15.9 & 17.7 & 0.129 & P00441 & 1 & 0.13 \\
\hline SOD2 & superoxide dismutase 2 , mitochondrial & 0.000 & 32.3 & 6.9 & 7.7 & 23.7 & 23.7 & 0.148 & P04179 & 3 & 0.23 \\
\hline TRA1 & $\begin{array}{l}\text { tumor rejection antigen (gp96) } 1 \text { (heat shock } \\
\text { protein gp96) }\end{array}$ & 0.008 & 9.1 & 4.7 & 4.7 & 90.4 & 95.0 & 0.043 & P14625 & 1 & 0.04 \\
\hline \multicolumn{12}{|c|}{ 4. Immune Response } \\
\hline GZMA & granzyme A precursor & 0.033 & 16.0 & 9.1 & 7.3 & 29.0 & 21.9 & 0.077 & P12544 & 1 & 0.08 \\
\hline HLA-B & major histocompatibility complex, class I, B & 0.130 & 12.0 & 6.1 & 7.8 & 40.5 & 31.2 & 0.040 & P01889 & 1 & 0.04 \\
\hline HLA-G & major histocompatibility complex, class I, G & 0.083 & 32.7 & 5.0 & 7.4 & 19.2 & 68.4 & 0.109 & P17693 & 1 & 0.11 \\
\hline IGHG2 & immunoglobulin heavy chain & 0.028 & 31.7 & 5.2 & 6.8 & 35.9 & 43.3 & 0.026 & P01859 & 2 & 0.16 \\
\hline TCRB & T cell receptor $B$ chain & 0.002 & 32.7 & 6.5 & 9.5 & 19.9 & 26.9 & 0.081 & P01850 & 2 & 0.10 \\
\hline \multicolumn{12}{|l|}{ 5. Apoptosis } \\
\hline CASP2 & caspase-2 precursor & 0.140 & 18.0 & 6.1 & 6.1 & 34.9 & 27.1 & 0.036 & P42575 & 2 & 0.04 \\
\hline CASP8 & caspase 8 & 0.100 & 12.0 & 5.7 & 6.4 & 30.7 & 26.7 & 0.061 & Q14790 & 1 & 0.06 \\
\hline CTSD & cathepsin D (lysosomal aspartyl protease) & 0.010 & 14.5 & 5.3 & 5.3 & 44.5 & 35.8 & 0.016 & P07339 & 3 & 0.02 \\
\hline \multicolumn{12}{|c|}{ 6. Cell Division and Proliferation } \\
\hline FGFR3 & fibroblast growth factor receptor 3 & 0.090 & 10.0 & 5.7 & 6.6 & 88.1 & 84.3 & 0.088 & P22607 & 1 & 0.09 \\
\hline $\mathrm{MLH} 1$ & DNA mismatch repair protein Mlh1 & 0.088 & 10.0 & 5.5 & 7.2 & 84.7 & 90.8 & 0.022 & P40692 & 1 & 0.02 \\
\hline \multicolumn{12}{|c|}{ 7. Cell Signaling and Control of Transcription and Translation } \\
\hline FOXP4 & forkhead box P4 isoform 1 & 0.086 & 24.8 & 4.5 & 7.8 & 16.2 & 80.8 & 0.014 & Q8IVH2 & 1 & 0.01 \\
\hline GJE1 & gap junction protein, epsilon 1, 29 kDa & 0.031 & 17.0 & 9.4 & 6.7 & 31.3 & 60.8 & 0.065 & Q8NFK1 & 2 & 0.08 \\
\hline PHB & prohibitin & 0.002 & 37.5 & 5.6 & 5.9 & 29.9 & 34.3 & 0.042 & P35232 & 2 & 0.06 \\
\hline TUFM & $\begin{array}{l}\text { elongation factor Tu, mitochondrial precursor } \\
\text { (EF-Tu) (P43) }\end{array}$ & 0.020 & 19.0 & 7.9 & 7.3 & 49.9 & 56.8 & 0.052 & P49411 & 3 & 0.15 \\
\hline \multicolumn{12}{|c|}{ 8. Other Functions } \\
\hline ALB & albumin precursor & 0.057 & 17.3 & 5.6 & 6.2 & 68.4 & 76.0 & 6.653 & P02768 & 5 & 6.65 \\
\hline ANXA5 & annexin $\mathrm{A} 5$ & 0.090 & 16.6 & 4.9 & 4.9 & 35.8 & 41.0 & 0.024 & P08758 & 1 & 0.02 \\
\hline ARFGAP1 & ADP-ribosylation factor GTPase activating protein 1 & 0.032 & 27.8 & 4.4 & 5.6 & 44.7 & 46.1 & 0.019 & Q8N6T3 & 1 & 0.02 \\
\hline C16orf49 & $\begin{array}{l}\text { chromosome } 16 \text { open reading frame } 49 \\
\text { (unknown gene product) }\end{array}$ & 0.020 & 25.2 & 5.6 & 4.9 & 35.7 & 43.8 & 0.026 & O75208 & 3 & 0.03 \\
\hline CA1 & carbonic anhydrase I & 0.001 & 38.8 & 6.6 & 7.4 & 28.8 & 32.2 & 0.019 & P00915 & 1 & 0.02 \\
\hline HBA1 & hemoglobin, $\alpha 1$ & 0.026 & 14.1 & 9.1 & 9.4 & 15.0 & 11.2 & 0.274 & P69905 & 2 & 0.29 \\
\hline HBB & hemoglobin, $B$ & 0.002 & 40.6 & 6.8 & 7.8 & 15.0 & 11.5 & 0.101 & P68871 & 2 & 0.20 \\
\hline IMMT & inner membrane protein, mitochondrial & 0.000 & 9.0 & 6.1 & 6.5 & 83.6 & 84.2 & 0.112 & Q16891 & 4 & 0.26 \\
\hline MB & myoglobin & 0.018 & 44.2 & 7.9 & 3.1 & 17.2 & 15.5 & 1.120 & P02144 & 7 & 3.79 \\
\hline MMP13 & matrix metalloproteinase 13 (collagenase 3 ) & 0.130 & 25.0 & 5.3 & 8.4 & 53.8 & 55.6 & 0.067 & P45452 & 3 & 0.13 \\
\hline NUP153 & nucleoporin $153 \mathrm{kDa}$ & 0.087 & 4.0 & 8.9 & 6.6 & 153.8 & 69.9 & 0.018 & P49790 & 1 & 0.02 \\
\hline RTN1 & reticulon 1 & 0.080 & 7.1 & 4.6 & 6.7 & 83.9 & 68.9 & 0.036 & Q16799 & 1 & 0.04 \\
\hline TF & transferrin & 0.040 & 7.9 & 7.1 & 7.0 & 79.3 & 82.4 & 0.336 & P02787 & 3 & 0.50 \\
\hline
\end{tabular}

${ }^{1}$ Expectation; ${ }^{2}$ coverage; ${ }^{3}$ normalized volume; ${ }^{4}$ number of spots identified as the same protein; ${ }^{5}$ total volume (total volume of all spots identified as the named protein). $(E)=$ experimental; $(T)=$ theoretical. 
belonged to a metabolic pathway. Most of the proteins identified in this group were enzymes or enzyme subunits related to energy metabolism, belonging to the glycolysis (5 proteins), $\beta$-oxidation ( 2 proteins), tricarboxylic acid cycle (7 proteins) and electron transport and oxidative phosphorylation (11 proteins) pathways. As examples, aconitase, fumarate hydratase, pyruvate dehydrogenase, and enolase play important roles in the tricarboxylic acid cycle and NADH dehydrogenase isoforms and cytochrome $\mathrm{c}$ reductase are subunits of the complexes from the respiratory chain. We also identified members of the energy transduction (creatine kinase) pathway; significantly, a high number of spots were identified as creatine kinase $\mathrm{M}$ isoforms (Table 1, Figure 1), which catalyzes the transphosphorylation reaction of mitochondrial ATP to phosphocreatine and ADP for cytoplasmic ATP synthesis (18). The creatine kinase pathway is an important complementary pathway under conditions of high ATP demand (19). We also identified other members of the lipid metabolism pathway, as well as three proteins involved in nucleotide/nucleic acid metabolism.

The identification of a significant number of proteins involved in stress (19\%), in the immune response (4\%) and in apoptotic (3\%) processes reinforces their importance in the pathogenesis of CCC. Among the stress proteins, the most frequently identified were heat shock proteins (HSP) and cognates. In the present study, 10 HSP were identified (isoforms of HSP27, HSP40, HSP60, HSP70, and HSP90). Crystallin is also a member of this protein superfamily (20). Oxidative stress-related proteins such as peroxiredoxin and both $\mathrm{Cu} / \mathrm{Zn}$ and $\mathrm{Mn}$ superoxide dismutases were also frequently identified; these proteins are antioxidants and protect the cell against free radicals, especially reactive oxygen intermediates (21).

Analysis of the proteomic profile of the CCC myocardium also identified proteins

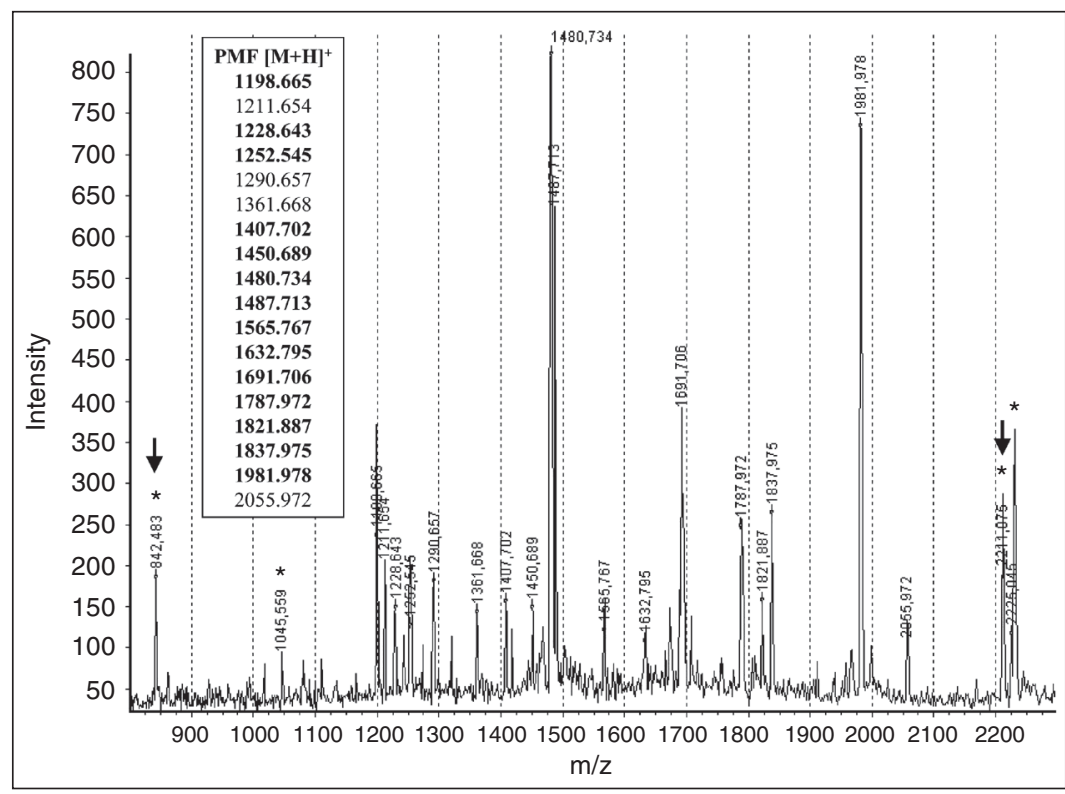

Figure 2. MALDI-ToF mass spectrum of the tryptic products from a spot. The peptide mass fingerprinting (PMF) list used for protein identification in the databanks is shown. *Trypsin autolysis peptides used for internal calibration. Peaks that appeared in all spectra were not included in the PMF list. In bold, the $\mathrm{m} / \mathrm{z}$ values from the PMF list that were matched with the identified protein from the database. This spectrum was identified as a heat shock $70-\mathrm{kDa}$ protein 8 isoform 2 (expectation: 0.003 , coverage: $37.1 \%$ ) using the non-redundant NCBI databank (Homo sapiens).

Table 2. Functional classification of the proteins identified in the myocardium from patients with chronic Chagas' disease cardiopathy.

\begin{tabular}{lr}
\hline Functional classification & $\%$ \\
\hline 1. Structural and contractile proteins & 19 \\
2. Metabolism & 38 \\
2.1. Glycolysis & 12 \\
2.2. Tricarboxylic acid cycle & 17 \\
2.3. Oxidative phosphorylation and electron transport & 26 \\
2.4. Energy transduction & 5 \\
2.5. Lipid metabolism/ B oxidation & 14 \\
2.6. Nucleotide and nucleic acid metabolism & 7 \\
2.7. Other metabolism proteins & 19 \\
3. Stress response & 20 \\
4. Immune response & 4 \\
5. Apoptosis & 3 \\
6. Cell division and proliferation & 2 \\
7. Cell signaling and control of transcription and translation & 4 \\
8. Other functions & 12
\end{tabular}

The functional classification of the identified proteins was adapted from the Gene Ontology classification using the FatiGO tool (http://fatigo.bioinfo.cipf.es). 
related to the immune system, such as immunoglobulins, $\mathrm{T}$ cell receptor $\beta$ chain, HLA class I molecule, and granzyme A. The expression of HLA class I and granzyme A is increased expression in the myocardium from CCC patients $(7,8)$. Granzyme A, a protein known to be produced by CD8+ cytotoxic $\mathrm{T}$ cells - which are highly abundant in CCC myocardium (22) - triggers apoptosis of target cells in the presence of CD8+ T cell cytotoxicity (23). Regarding proteins related to the apoptotic process, we found caspases 2 and 8 in CCC heart whose sequential activation plays a central role in cell apoptosis (24).

Proteins related to cell division and proliferation and related to cell signaling and to the control of transcription and translation were 2 and $4 \%$ of the identified proteins, respectively. Some of these proteins were: prohibitin, which plays a role in regulating proliferation and inhibits DNA synthesis (25), Forkhead box P4 isoform 1, which is a transcriptional repressor that has been involved in cardiac morphogenesis (26), and protein disulfide isomerase-associated 3 , which is classified as a signal transduction protein, but which could also be classified as an electron transport protein and plays a role in the oxidative process (27).

Several spots from the CCC gels, with different $\mathrm{pI}$ and mass, were identified by $\mathrm{PMF}$ as the same protein, such as aconitase, creatine kinase $\mathrm{M}$ chain, and heat shock 70-

Figure 3. Validation of selected proteins by Western blot analysis of 6 myocardium samples from patients with chronic Chagas' disease cardiopathy. $A$, Identification of HSP60 (61 kDa), PDI $(\sim 55 \mathrm{kDa})$ and CuZn SOD ( 23 $\mathrm{kDa}$ ), which are involved in the oxidative stress process. $B$, Identification of apoptotic proteins, $\alpha$ and $B$ caspase $8(\sim 58 \mathrm{kDa})$ and caspase $2(48 \mathrm{kDa})$. The processed form of caspase $8(\sim 26$ $\mathrm{kDa})$ was also identified. HSP $=$ heat shock protein; $\mathrm{PDI}=$ protein disulfide isomerase; SOD = superoxide dismutase.
A

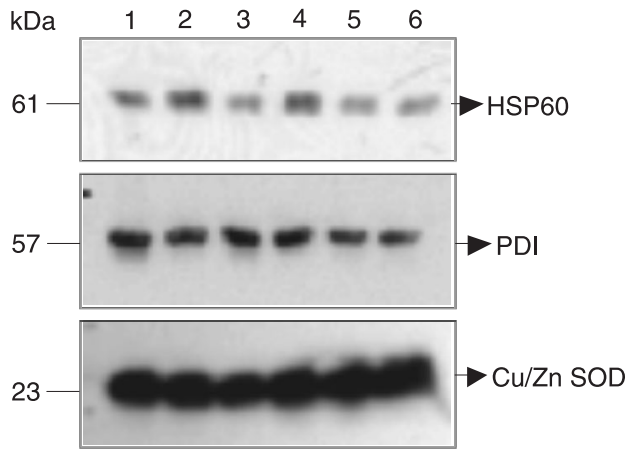

$\mathrm{kDa}$ protein, which were identified in 7,8 , and 6 spots, respectively. Structural and contractile proteins, such as several forms of actin (ACTA1, ACTA2, ACTC, ACTG2, ACTN2), desmin, myosin (MYL3 and MYL7), and vimentin were also identified with different pI values. These could be a result of post-translational modifications such as oxidation, phosphorylation, acetylation, or other processes. Some proteins presented differences in both mass and $\mathrm{pI}$ compared to the Swiss-Prot database, such as class I major histocompatibility complex G (HLA-G), which shows a difference between the experimental and theoretical value of $2.4 \mathrm{pI}$ units, while the theoretical mass was $49 \mathrm{kDa}$ less than the experimental mass. The same occurred with immunoglobulin heavy chain (IGHG2) which showed a difference between the experimental and theoretical value of $1.6 \mathrm{pI}$ units, and the theoretical mass was $7.4 \mathrm{kDa}$ less than the experimental mass.

In order to validate the mass spectrometric identification of proteins, we selected 5 representative identified proteins (caspase 8 , caspase 2 , hsp60, $\mathrm{Cu} / \mathrm{Zn}$ superoxide dismutase, and protein disulfide isomerase 3) for further analysis by immunoblot with the aid of specific monoclonal antibodies, in tissue samples from $6 \mathrm{CCC}$ patients (Figure $3)$. We identified protein bands of the expected molecular weights reacting with the 5 specific antibodies in all samples tested, con-

B

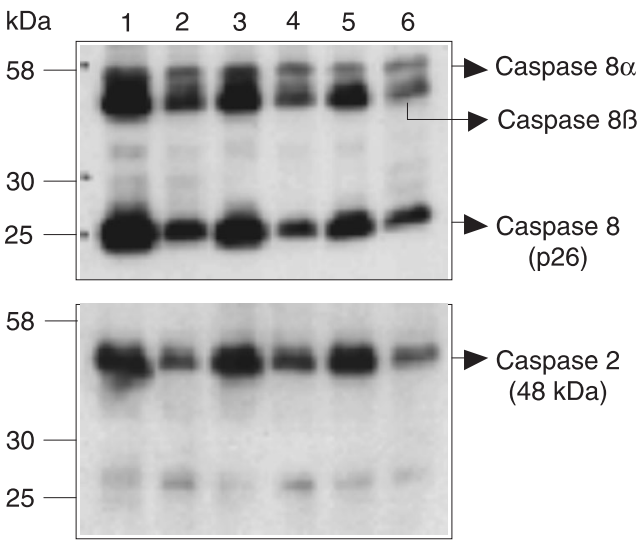


firming the existence of proteins identified by PMF. Furthermore, we observed the processed forms of caspase 8 , indicating its possible activation.

\section{Discussion}

We describe the protein profile of the myocardium from patients with chronic Chagas' disease cardiomyopathy. To our knowledge, although data from several differential proteome analyses in myocardial samples are available in the literature, the present study is the first report on the myocardial protein profile of CCC patients. Besides identifying the abundant structural and contractile myocardial proteins, this approach allowed us to identify proteins not expected to be found in normal myocardium, such as stress and oxidative stress proteins, apoptosis effector molecules and proteins involved in the immune response.

Structural proteins such as sarcomeric actomyosin complex constituents, as well as cytoskeletal proteins, were frequently identified, as expected, since sarcomeric contractile proteins are the most abundant proteins in the myocardium. Among the structural proteins, the atrial isoform of MYL4 was identified in the $\mathrm{CCC}$ ventricle samples. Ventricular expression of atrial proteins has been observed in cardiac hypertrophy and has been ascribed to reversal of myocytes to an embryonic program of gene transcription (16). Vimentin, highly expressed in fibroblasts, was also identified, in agreement with the intense fibrotic process known to occur in CCC heart tissue (17). We could also identify matrix metalloproteinase 13 (MMP13), also called collagenase- 3 , which is expressed by different cell types including stromal fibroblasts. MMP13 degrades collagens I, II, III, gelatin, fibronectin, laminin, and tenascin (28). Other MMP such as MMP9 (29) has been reported to play a role in cardiac remodeling. Thus, we may suggest that MMP13 could also be involved in the in- tense remodeling that occurs in the $\mathrm{CCC}$ myocardium.

The finding that $27 \%$ of the identified proteins belong to the different pathways of energy metabolism that directly participate in the generation of ATP (glycolysis, Boxidation, tricarboxylic acid cycle, oxidative phosphorylation, and creatine kinase system) (Table 1) is consistent with the fact that the heart requires significant energy for normal cardiac function (19,30). Moreover, most of the cardiac diseases in which mechanical dysfunction development occurs are associated with a deficit in energy production, often generated by disturbances in one or more metabolic steps of the ATP production pathways (19).

A significant number of HSP and cognates (40 spots, 13 distinct HSP) were identified in CCC myocardium. Transient HSP synthesis protects cellular homeostasis after exposure to heat and to a wide spectrum of stressful and potentially deleterious stimuli $(31,32)$. Accumulating evidence has implicated HSPs as mediators of myocardial protection, particularly in experimental models of ischemia and reperfusion injury (31). Different protective functions have been attributed to HSPs, including repairing ion channels, restoring redox balance, interacting with nitric oxide-induced protection, inhibiting proinflammatory cytokines, and preventing activation of the apoptosis pathway (31). The significant number of spots identified as HSPs in the CCC samples supports their role in myocardial protection. We could identify 12 spots as isoforms of HSP70, which is expressed at low levels in normal tissue and is rapidly induced in response to ischemic stress (33). The increase in myocardial HSP60 production has also been associated with the development of chronic heart failure (32).

Multiple spots were identified as oxidative stress-related proteins, such as peroxiredoxin and both $\mathrm{Cu} / \mathrm{Zn}$ and $\mathrm{Mn}$ superoxide dismutase. Studies on animal models have 
suggested that a chronic increase in oxidative stress in the myocardium, possibly due to impairment of superoxide dismutase and other antioxidant pathways, could contribute to myocardial remodeling and failure (34). A murine model of $T$. cruzi infection showed that the progressive severity of disease of infected mice is associated with increased oxidative damage in the myocardium, which seems to result from enhanced oxidant production coupled with diminishing antioxidant defense (35).

Proteins that play a role in immune processes were also detected in CCC myocardium. T cell receptor and immunoglobulin are consistent with the mononuclear infiltrate that is a hallmark of CCC. Moreover, these findings are technically interesting, since 2D gel electrophoresis/PMF proteomic analysis is expected to identify only the most abundantly expressed proteins from the cardiac cells, rather than proteins from a comparatively small infiltrating inflammatory cell population observed in CCC myocardium. We also identified granzyme $\mathrm{A}$, that initiates an alternative pathway for granule-mediated apoptosis (36) and is one of the three key components of the lytic granules of $\mathrm{CD} 8+\mathrm{T}$ cells, which constitute the majority of the mononuclear infiltrate (22). However, one cannot rule out the possibility that these findings are due to blood contamination.

In our profile experiment we found caspases 2 and 8 . Caspase 8 plays a role in CD95- and tumor necrosis factor-induced cell death (24). Apoptosis has been associated with heart damage in numerous diseases, such as viral infection, myocardial infarction, diabetes, and regression of hypertrophy $(37,38)$. Apoptosis assessed by the TUNEL method has been shown in CCC, and myocardial cell loss by apoptosis may contribute to heart failure (39). This is reinforced by the identification of activated caspase 8 forms in all 6 CCC myocardium samples (Figure 3).

The observation of several spots identi- fied by PMF as the same protein, but showing distinct pI's may represent different isoforms of the protein, post-translational modifications or technical artifacts of the 2-D methodology. Post-translational modifications such as phosphorylation or acetylation may be visualized on 2-D electrophoresis gels as a "train of spots" differing only by their pI, and have been observed previously by other investigators. These protein modifications were not characterized in the present study, but such trains of spots were observed in some structural and contractile proteins such as several forms of actin (ACTA1, ACTA2, ACTC, ACTG2, ACTN2), desmin, MYL3 and MYL7, and vimentin, that were identified with different $\mathrm{pI}$ values. Other spots from the CCC gels, with different $\mathrm{pI}$ and mass, were also identified as the same protein, such as aconitase, creatine kinase $\mathrm{M}$ chain, and heat shock $70-\mathrm{kDa}$ protein that were identified in 7, 8, and 6 spots, respectively. The reason for this large number of isoforms shown by some proteins is not known. Literature data suggest that a subset of proteins from cardiac mitochondria appear to be susceptible to double oxidation of their tryptophan residues (40). The fact that some proteins presented differences between experimental and theoretical mass and pI (e.g., HLA-G and IGHG2) could be due to the matching with sequence fragments in the databanks, rather than complete protein sequences.

Several isoforms of the same protein showing distinct experimental mass and $\mathrm{pI}$ may also represent hydrolytic fragments formed during breakdown of the parent molecules in vivo or in vitro, with oxidation forms resulting from the 2-D gel processing and precursors arising during protein assembly. Another limitation of the present study was that the proteins identified here may not originate from the cardiac tissue itself. As in most mammalian organs, the involvement of vascular tissue needs to be considered, and this may explain the identification of 
smooth muscle forms of actin. Blood-derived proteins were also found, including albumin, hemoglobin and transferrin.

Proteomic analysis including 2-D gel electrophoresis and PMF has allowed us to identify 246 matched protein spots, representative of 112 distinct proteins, in myocardial tissue from end-stage CCC patients. This paper has described for the first time a significant expression of oxidative stress and apoptosis-related proteins in CCC heart tissue, suggesting the possible participation of such processes in CCC pathogenesis, a subject that is currently under investigation.

\section{Acknowledgments}

The authors thank Dr. Mario Palma (Universidade Estadual de São Paulo, Rio Claro, SP, Brazil) and Drs. Simone Fonseca, Angelina Bilate and Kellen Fae (Universidade de São Paulo, São Paulo, SP, Brazil) for critical readings and discussion.

\section{References}

1. Morel CM, Lazdins J. Chagas disease. Nat Rev Microbiol 2003; 1 : 14-15.

2. Bestetti RB, Muccillo G. Clinical course of Chagas' heart disease: a comparison with dilated cardiomyopathy. Int J Cardiol 1997; 60: 187-193.

3. Freitas HF, Chizzola PR, Paes AT, Lima AC, Mansur AJ. Risk stratification in a Brazilian hospital-based cohort of 1220 outpatients with heart failure: role of Chagas' heart disease. Int J Cardiol 2005; 102: 239-247.

4. Higuchi ML, De Morais CF, Pereira Barreto AC, Lopes EA, Stolf N, Bellotti $G$, et al. The role of active myocarditis in the development of heart failure in chronic Chagas' disease: a study based on endomyocardial biopsies. Clin Cardiol 1987; 10: 665-670.

5. Reis MM, Higuchi ML, Benvenuti LA, Aiello VD, Gutierrez PS, Bellotti $G$, et al. An in situ quantitative immunohistochemical study of cytokines and IL-2R in chronic human chagasic myocarditis: correlation with the presence of myocardial Trypanosoma cruzi antigens. Clin Immunol Immunopathol 1997; 83: 165-172.

6. Abel LC, Rizzo LV, lanni B, Albuquerque F, Bacal F, Carrara D, et al. Chronic Chagas' disease cardiomyopathy patients display an increased IFN-gamma response to Trypanosoma cruzi infection. $J$ Autoimmun 2001; 17: 99-107.

7. Reis DD, Jones EM, Tostes S Jr, Lopes ER, Gazzinelli G, Colley $D G$, et al. Characterization of inflammatory infiltrates in chronic chagasic myocardial lesions: presence of tumor necrosis factoralpha+ cells and dominance of granzyme A+, CD8+ lymphocytes. Am J Trop Med Hyg 1993; 48: 637-644.

8. Reis DD, Jones EM, Tostes S, Lopes ER, Chapadeiro E, Gazzinelli $\mathrm{G}$, et al. Expression of major histocompatibility complex antigens and adhesion molecules in hearts of patients with chronic Chagas' disease. Am J Trop Med Hyg 1993; 49: 192-200.

9. Cunha-Neto E, Kalil J. Heart-infiltrating and peripheral T cells in the pathogenesis of human Chagas' disease cardiomyopathy. Autoimmunity 2001; 34: 187-192.

10. Teixeira MM, Gazzinelli RT, Silva JS. Chemokines, inflammation and Trypanosoma cruzi infection. Trends Parasitol 2002; 18: 262265.

11. Gomes JA, Bahia-Oliveira LM, Rocha MO, Martins-Filho OA, Gazzinelli G, Correa-Oliveira R. Evidence that development of severe cardiomyopathy in human Chagas' disease is due to a Th1- specific immune response. Infect Immun 2003; 71: 1185-1193.

12. Cunha-Neto E, Dzau VJ, Allen PD, Stamatiou D, Benvenutti L, Higuchi ML, et al. Cardiac gene expression profiling provides evidence for cytokinopathy as a molecular mechanism in Chagas' disease cardiomyopathy. Am J Pathol 2005; 167: 305-313.

13. Arrell DK, Neverova I, Van Eyk JE. Cardiovascular proteomics: evolution and potential. Circ Res 2001; 88: 763-773.

14. Sutton CW, Pemberton KS, Cottrell JS, Corbett JM, Wheeler $\mathrm{CH}$, Dunn MJ, et al. Identification of myocardial proteins from two-dimensional gels by peptide mass fingerprinting. Electrophoresis 1995; 16: 308-316.

15. Smith PK, Krohn RI, Hermanson GT, Mallia AK, Gartner FH, Provenzano MD, et al. Measurement of protein using bicinchoninic acid. Anal Biochem 1985; 150: 76-85.

16. Schwartz K, Boheler KR, de la Bastie D, Lompre AM, Mercadier JJ. Switches in cardiac muscle gene expression as a result of pressure and volume overload. Am J Physiol 1992; 262: R364-R369.

17. Rossi MA. The pattern of myocardial fibrosis in chronic Chagas' heart disease. Int J Cardiol 1991; 30: 335-340.

18. Wallimann T. Dissecting the role of creatine kinase. Curr Biol 1994; 4: $42-46$.

19. Carvajal K, Moreno-Sanchez R. Heart metabolic disturbances in cardiovascular diseases. Arch Med Res 2003; 34: 89-99.

20. Ingolia TD, Craig EA. Four small Drosophila heat shock proteins are related to each other and to mammalian alpha-crystallin. Proc Natl Acad Sci U S A 1982; 79: 2360-2364.

21. Nordberg J, Arner ES. Reactive oxygen species, antioxidants, and the mammalian thioredoxin system. Free Radic Biol Med 2001; 31: 1287-1312.

22. Higuchi MD, De Brito T, Reis MM, Bellotti G, Pereira-Barretto AC, Pileggi F. Correlation between $T$. cruzi parasitism and myocardial inflammatory infiltrate in human chronic chagasic myocarditis: light microscopy and immunohistochemical findings. Cardiovasc Pathol 1993; 2: 101-105.

23. Pardo J, Bosque A, Brehm R, Wallich R, Naval J, Mullbacher A, et al. Apoptotic pathways are selectively activated by granzyme $A$ and/ or granzyme B in CTL-mediated target cell lysis. J Cell Biol 2004; 167: 457-468.

24. Cohen GM. Caspases: the executioners of apoptosis. Biochem $J$ 1997; 326 (Pt 1): 1-16. 
25. Mishra S, Murphy LC, Nyomba BL, Murphy LJ. Prohibitin: a potential target for new therapeutics. Trends Mol Med 2005; 11: 192-197.

26. Li S, Zhou D, Lu MM, Morrisey EE. Advanced cardiac morphogenesis does not require heart tube fusion. Science 2004; 305: 16191622.

27. Janiszewski M, Lopes LR, Carmo AO, Pedro MA, Brandes RP, Santos $C X$, et al. Regulation of $\mathrm{NAD}(\mathrm{P}) \mathrm{H}$ oxidase by associated protein disulfide isomerase in vascular smooth muscle cells. J Biol Chem 2005; 280: 40813-40819.

28. Creemers EE, Cleutjens JP, Smits JF, Daemen MJ. Matrix metalloproteinase inhibition after myocardial infarction: a new approach to prevent heart failure? Circ Res 2001; 89: 201-210.

29. Ducharme A, Frantz S, Aikawa M, Rabkin E, Lindsey M, Rohde LE, et al. Targeted deletion of matrix metalloproteinase- 9 attenuates left ventricular enlargement and collagen accumulation after experimental myocardial infarction. J Clin Invest 2000; 106: 55-62.

30. Corbett JM, Why HJ, Wheeler CH, Richardson PJ, Archard LC, Yacoub $\mathrm{MH}$, et al. Cardiac protein abnormalities in dilated cardiomyopathy detected by two-dimensional polyacrylamide gel electrophoresis. Electrophoresis 1998; 19: 2031-2042.

31. Delogu G, Signore M, Mechelli A, Famularo G. Heat shock proteins and their role in heart injury. Curr Opin Crit Care 2002; 8: 411-416.

32. Tanonaka K, Yoshida H, Toga W, Furuhama K, Takeo S. Myocardial heat shock proteins during the development of heart failure. Biochem Biophys Res Commun 2001; 283: 520-525.
33. Marber MS, Mestril R, Chi SH, Sayen MR, Yellon DM, Dillmann WH. Overexpression of the rat inducible $70-\mathrm{kD}$ heat stress protein in a transgenic mouse increases the resistance of the heart to ischemic injury. J Clin Invest 1995; 95: 1446-1456.

34. Dhalla AK, Hill MF, Singal PK. Role of oxidative stress in transition of hypertrophy to heart failure. J Am Coll Cardiol 1996; 28: 506-514.

35. Wen JJ, Vyatkina G, Garg N. Oxidative damage during chagasic cardiomyopathy development: role of mitochondrial oxidant release and inefficient antioxidant defense. Free Radic Biol Med 2004; 37 : 1821-1833.

36. Shresta S, Graubert TA, Thomas DA, Raptis SZ, Ley TJ. Granzyme A initiates an alternative pathway for granule-mediated apoptosis. Immunity 1999; 10: 595-605.

37. Frustaci A, Kajstura J, Chimenti C, Jakoniuk I, Leri A, Maseri A, et al. Myocardial cell death in human diabetes. Circ Res 2000; 87: 11231132.

38. Freude B, Masters TN, Kostin S, Robicsek F, Schaper J. Cardiomyocyte apoptosis in acute and chronic conditions. Basic Res Cardiol 1998; 93: 85-89.

39. Tostes S Jr, Bertulucci Rocha-Rodrigues D, de Araujo Pereira G, Rodrigues V Jr. Myocardiocyte apoptosis in heart failure in chronic Chagas' disease. Int J Cardiol 2005; 99: 233-237.

40. Taylor SW, Fahy E, Murray J, Capaldi RA, Ghosh SS. Oxidative post-translational modification of tryptophan residues in cardiac mitochondrial proteins. J Biol Chem 2003; 278: 19587-19590. 